\title{
Editorial
}

\section{Role of Genetics in Musculoskeletal Injuries: The Unsolved Jigsaw Puzzle}

'The more I learn, the more I realize how much I do not know' - Albert Einstein

This quote succinctly summarizes our current stand on genetic research in musculoskeletal injuries. Although, our understanding of clinical aspects of common musculoskeletal injuries has increased by leaps and bounds in the past century, the knowledge of the biomolecular mechanisms of these injuries is still primitive at its best. Tremendous progress has been made in identifying genetic factors that increase susceptibility to cancer and other diseases, which have helped identify potential prevention and treatment options. However, only few researchers have focussed on the genetic risk factors predisposing to musculoskeletal injuries. Studies are now trickling in from different parts of the globe identifying genetic mutations, which may be one of the multifactorial issues at play and which may help us predict soft tissue injury risk for specific individuals or population groups.

One must first understand the spectrum of connective tissue disorders that can have a genetic component. On one end of the spectrum are the Mendelian inherited orthopedic conditions like Ehlers-Danlos syndrome, Marfan's syndrome, osteogenesis imperfecta, skeletal dysplasias, neurocutaneous syndromes, and so on where the severity of the disease is directly determined by the genetic variations. However, on the other end of the spectrum are multifactorial disorders with a complex, non-Mendelian inheritance wherein the development and severity of the condition is determined by complex gene-gene and gene-environment interactions. Osteoarthritis, osteoporosis, tendon and ligament injuries, and so on are examples of conditions which fall under this side of the spectrum and they constitute a large bulk of musculoskeletal problems today.

We now realized that many common musculoskeletal injuries like anterior cruciate ligament (ACL) tear, Achilles tendinopathy and rotator cuff tears may be multifactorial in origin, with the actual injury being just an inciting factor. Genetic risk factors have been identified of late and some research focus on single nucleotide polymorphisms (SNPs) is emerging, with recent research being done at the PGIMER also. ${ }^{1}$

Familial predisposition to an ACL tear was reported way back in $1994,{ }^{2}$ but the first specific gene polymorphisms which may predispose an individual to an ACL tear were identified only in 2008. In two independent studies in different Caucasian populations, ${ }^{3,4}$ the thymidine-thymidine (TT) genotype of the specific protein 1 (Sp1) binding site polymorphism of the COL1A1 gene was shown to be underrepresented in patients with an ACL tear [COL1A1 gene encodes for $\alpha 1$ (1) of type 1 collagen which is the

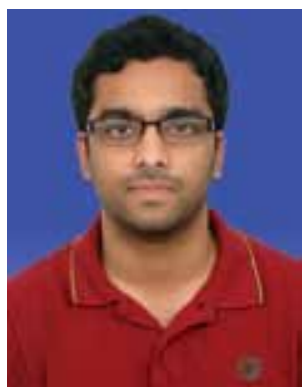

Rakesh John

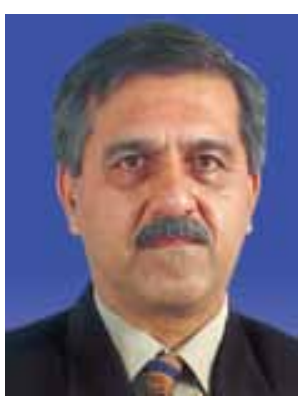

Mandeep S Dhillon

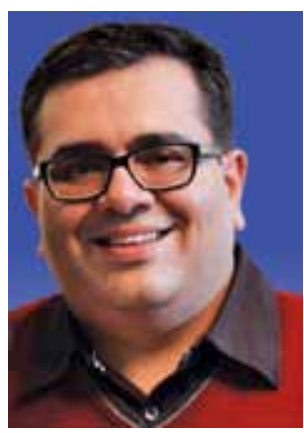

Sharad Prabhakar major building block of the collagen fibrils]. Interestingly, the Sp1 binding site has previously also been associated with various other connective tissue disorders like myocardial infarction, lumbar disk disease, stress urinary incontinence and most notably osteoporosis. A few recent studies have also linked spontaneous shoulder dislocations and full thickness rotator cuff tears with polymorphisms in COL1A1 gene.

Although the sample sizes were small, it was observed that polymorphisms in COL12A1 and COL5A1 genes were associated with an increased risk of ACL tear in females. An association of ACL tear with the chromosomal region 11q22, which is home to many matrix metalloproteinase genes (MMPs), has also been proposed. AG and GG genotypes of MMP1 gene were shown to be significantly underrepresented in ACL tear patients. Recently, Bell et al showed that SNPs of COL5A1 and COL12A1 previously associated with ACL tear were also positively associated with increased joint laxity. ${ }^{6}$

In a study conducted very recently in our own institute, ${ }^{1}$ which happens to be the first study in the world in a non-Caucasian population, we noted a positive association between ACL tear and polymorphisms in COL12A1 gene. However, no association was found between COL1A1 polymorphisms and ACL tear in the Indian population. We also went one step ahead of other research groups by analyzing the protein expression of these gene polymorphisms by enzyme-linked immunosorbent assay (ELISA) testing.

A look at the literature on genetic risk factors of yet another common orthopedic soft tissue disorder, Achilles tendinopathy, reveals an association of tenascin C (TNC), COL5A1 and MMP3 gene polymorphisms with chronic cases. The sequence variant in TNC gene is also significantly associated with spontaneous Achilles tendon ruptures. It is to be noted that Achilles tendinopathy was also one of the many disorders which was associated with ABO blood group (O negative blood group was associated with an increased tendency to Achilles tendon tear, whereas A blood group was associated with a reduced risk). Recent studies have, however, failed to substantiate the association of $\mathrm{ABO}$ blood group with Achilles tendon pathology. What is interesting to note here is that the ABO gene (located on long arm of chromosome 9) is in close proximity to the TNC and COL5A1 genes? Thus, ABO blood group might actually be a biochemical marker for the actual genes involved in Achilles tendinopathy. ${ }^{5}$ 
Researchers have likened the current status of genetics in musculoskeletal injuries to a complicated jigsaw puzzle, where only a few pieces have been identified and efforts are being made to see where these pieces fit and how they are related to each other. Though, the recent advances made in genetic research have not affected our clinical management of these conditions, this will certainly change in the future. The ultimate goal of these studies is not just genetic testing to identify predisposed individuals/ athletes but to roll it into a complex multifactorial disease model. This model can then be used to ameliorate the effects of nonmodifiable risk factors (i.e. genetic risk factors) by modifiable risk factors through reduced exposure to specific risks and personalizing training programs.

Future work should be directed at testing larger cohorts in different ethnic populations. Multidisciplinary research must be encouraged to link genetics to cell biology to tissue function to whole body function. Epigenetics (study of influence of environmental factors on gene expression) is another exciting field of research that awaits exploration. Although not in the near future, gene therapy is expected to follow in the footsteps of the march of genetic research, thus opening up exciting new possibilities and treatments in the not so distant future!

\section{REFERENCES}

1. John R. Association of gene polymorphisms in COL1A1 and COL12A1 with ACL tears: a study in the Indian population (thesis). PGIMER; Chandigarh 2013.

2. Harner CD, Paulos LE, Greenwald AE, Rosenberg TD, Cooley VC. Detailed analysis of patients with bilateral anterior cruciate ligament injuries. Am J Sports Med 1994 Jan-Feb;22(1):37-43.

3. Khoschnau S, Melhus H, Jacobson A, Rahme H, Bengtsson H, Ribom E, Grundberg E, Mallmin H, Michaëlsson K. Type I collagen alpha1 Sp1 polymorphism and the risk of cruciate ligament ruptures or shoulder dislocations. Am J Sports Med 2008 Dec;36(12):2432-2436.

4. Posthumus M, September AV, Keegan M, O'Cuinneagain D, Van der Merwe W, Schwellnus MP, Collins M. Genetic risk factors for anterior cruciate ligament ruptures: COL1A1 gene variant. Br J Sports Med 2009 May;43(5):352-356.

5. Jozsa L, Balint JB, Kannus P, Reffy A, Barzo M. Distribution of blood groups in patients with tendon rupture. J Bone Joint Surg Br 1989 Mar; 71(2):272-274.

6. Bell R, Shultz SJ, Wideman L, Henrich VC. Collagen gene variants previously associated with anterior cruciate ligament injury risk are also associated with joint laxity. Sports Health 2012;4:312-318.

Rakesh John MS

Senior Resident, Department of Orthopedics Postgraduate Institute of Medical Education and Research, Chandigarh, India e-mail: rakeshjohn23@gmail.com

Mandeep S Dhillon MS FAMS FRCS Professor, Department of Orthopedics Postgraduate Institute of Medical

Education and Research, Chandigarh, India e-mail:drdhillon@gmail.com

Sharad Prabhakar Assistant Professor, Department of Orthopedics Postgraduate Institute of Medical Education and Research, Chandigarh, India 